\title{
DOWNHILL DOMINATION IN GRAPHS
}

\author{
Teresa W. Haynes \\ Department of Mathematics and Statistics \\ East Tennessee State University \\ Johnson City, TN 37614, USA \\ e-mail: haynes@etsu.edu \\ Stephen T. Hedetniemi ${ }^{1}$ \\ School of Computing \\ Clemson University \\ Clemson, SC 29634, USA
}

e-mail: hedet@clemson.edu

AND

JESSIE D. JAMIESON ${ }^{2}$

William B. JAMIESON ${ }^{2}$

Department of Mathematics University of Nebraska-Lincoln Lincoln, NE 68588, USA

e-mail: jdjamieson@huskers.unl.edu wjamieson@huskers.unl.edu

\begin{abstract}
A path $\pi=\left(v_{1}, v_{2}, \ldots, v_{k+1}\right)$ in a graph $G=(V, E)$ is a downhill path if for every $i, 1 \leq i \leq k, \operatorname{deg}\left(v_{i}\right) \geq \operatorname{deg}\left(v_{i+1}\right)$, where $\operatorname{deg}\left(v_{i}\right)$ denotes the degree of vertex $v_{i} \in V$. The downhill domination number equals the minimum cardinality of a set $S \subseteq V$ having the property that every vertex $v \in V$ lies on a downhill path originating from some vertex in $S$. We investigate downhill domination numbers of graphs and give upper bounds. In particular, we show that the downhill domination number of a graph is at most half its order, and that the downhill domination number of a tree is at most one third its order. We characterize the graphs obtaining each of these bounds.
\end{abstract}

\footnotetext{
${ }^{1}$ Professor Emeritus.

${ }^{2}$ Work completed while attending East Tennessee State University.
} 
Keywords: downhill path, downhill domination number.

2010 Mathematics Subject Classification: 05C69, 05C38.

\section{REFERENCES}

[1] T.W. Haynes, S.T. Hedetniemi, J. Jamieson and W. Jamieson, Downhill and uphill domination in graphs, submitted for publication (2013).

[2] P. Hall, On representation of subsets, J. London Math. Soc. 10 (1935) 26-30.

[3] T.W. Haynes, S.T. Hedetniemi and P.J. Slater, Fundamentals of Domination in Graphs (Marcel Dekker, 1998).

[4] J.D. Hedetniemi, S.M. Hedetniemi, S.T. Hedetniemi and T. Lewis, Analyzing graphs by degrees, AKCE Int. J. Graphs Comb., to appear.

[5] O. Ore, Theory of Graphs (Amer. Math. Soc. Colloq. Publ. 38, 1962).

Received 8 April 2013

Revised 9 September 2013

Accepted 9 September 2013 